\title{
Correlates of meeting the combined and independent aerobic and strength exercise guidelines in hematologic cancer survivors
}

\author{
James R. Vallerand', Ryan E. Rhodes², Gordon J. Walker ${ }^{1}$ and Kerry S. Courneya ${ }^{1 *}$
}

\begin{abstract}
Background: Most previous research on the correlates of physical activity has examined the aerobic or strength exercise guidelines separately. Such an approach does not allow an examination of the correlates of meeting the combined guidelines versus a single guideline, or one guideline versus the other. Here, we report the prevalence and correlates of meeting the combined and independent exercise guidelines in hematologic cancer survivors (HCS).

Methods: In a population-based, cross-sectional survey of 606 HCS from Alberta, Canada using a mailed questionnaire, we obtained separate assessments of aerobic and strength exercise behaviors, as well as separate assessments for motivations, regulations, and reflective processes using the multi-process action control framework (M-PAC).

Results: Overall, 22\% of HCS met the combined exercise guideline, 22\% met aerobic-only, 10\% met strength-only, and $46 \%$ met neither exercise guideline. HCS were more likely to meet the combined guideline over the aerobic-only guideline if they had no children living at home, and over both the aerobic and strength-only guidelines if they had completed university. As hypothesized, those meeting the combined guideline also had a more favorable strengthspecific M-PAC profile (i.e., motivations, regulations, and reflective processes) than those meeting the aerobic-only guideline, and a more favorable aerobic-specific M-PAC profile than those meeting the strength-only guideline. Interestingly and unexpectedly, HCS meeting the combined guidelines also reported significantly greater aerobicspecific perceived control, planning, and obligation/regret than those meeting the aerobic-only guideline, and greater strength-specific perceived control, planning, and obligation/regret than those meeting the strength-only guideline.

Conclusions: Few HCS are meeting the combined exercise guidelines. M-PAC based variables are strong correlates of meeting the combined guidelines compared to aerobic or strength only guidelines. Strategies to help HCS meet the combined guidelines may need to promote more favorable behavioral regulations and reflective processes for both types of exercise rather than just the type of exercise in which HCS are deficient.
\end{abstract}

Keywords: Physical activity, Multi-process action control framework, M-PAC, Intention-behavior gap

\section{Background}

To improve cancer survivors' physical functioning and quality of life $[1,2]$, the American College of Sports Medicine (ACSM) recommends that survivors accumulate a weekly total of at least $150 \mathrm{~min}$ of moderate-tovigorous aerobic exercise (aerobic guideline), and two weekly sessions of strength training that target the major muscle groups (strength guideline) [3]. Complying with

\footnotetext{
*Correspondence: kerry.courneya@ualberta.ca

${ }^{1}$ Faculty of Physical Education and Recreation, University of Alberta,

Edmonton, AB, Canada

Full list of author information is available at the end of the article
}

this combined guideline serves as the optimal scenario for cancer survivors, as it affords them the unique benefits of both aerobic and strength exercise guidelines (e.g., cardiovascular health, body composition, physical functioning). Thus, understanding the determinants of the combined guideline is critical to the health of cancer survivors.

Previous research on the correlates of exercise has generally reported the correlates of "physical activity," with more recent efforts detailing the correlates separately for aerobic and strength exercise $[4,5]$. Crawford and colleagues have recently argued that the correlates 
of meeting the combined guideline may be different than a single guideline, and different strategies may be needed to motivate individuals to perform the combined guideline if they already meet one guideline or neither [6]. To explore these concepts, researchers need to examine the correlates of aerobic and strength exercise simultaneously. In an important first step, Crawford and colleagues followed this approach using a dataset of demographic and clinical variables, but these variables did not meaningfully distinguish between survivors meeting the combined guideline versus either single guideline [6]; prompting them to suggest that exercise-specific social cognitive variables may better differentiate these groups.

The purpose of the present study is to examine the correlates of meeting the combined and independent exercise guidelines in a population-based sample of hematologic cancer survivors (HCS). In our recent population-based survey of over 600 Albertan HCS, we examined their aerobic and strength exercise participation and motivations $[7,8]$, and focused our examination around a theoretical framework (the multi-process action control framework; M-PAC) which explicitly accounts for motivational (e.g., attitude, perceived control), regulatory (e.g., planning), and reflective (e.g., habit, identity) differences that characterize the gap existing between intention and behavior (known as the intention-behavior gap; I-B Gap) $[9,10]$. A particular strength of our study was that we assessed M-PAC based variables separately for both aerobic and strength exercise. In line with previous research, however, we reported the prevalence and correlates of aerobic and strength exercise separately. We now believe that a more informative approach would be to consider the correlates of both guidelines simultaneously.

We organized our hypotheses into themes: "traditional" and "novel." Our traditional hypotheses involved comparing demographic, cancer, and M-PAC based variables (i.e., motivational processes, behavioral regulations, and reflective processes) of HCS meeting the three exercise guidelines (combined, aerobic-only, and strengthonly) versus neither guideline. Our novel hypotheses involved comparing these same variables between each of the three exercise guidelines. Our traditional hypotheses were that key demographic and cancer-specific variables would differentiate HCS meeting either of the three exercise guidelines versus those meeting neither. Regarding the M-PAC based variables, we made the traditional hypotheses that compared to HCS meeting neither guideline, those meeting the combined or aerobic guidelines would have a more favorable aerobicspecific M-PAC profile, and those meeting the combined or strength guidelines would have a more favorable strength-specific M-PAC profile. In terms of novel hypotheses, we hypothesized that key demographic and cancer-specific variables would also differentiate HCS meeting the combined guidelines versus those meeting the aerobic-only and strength-only guidelines, and may even distinguish those meeting aerobic-only versus strength-only. We also made the novel hypotheses that those meeting the combined guideline would have a more favorable strength-specific M-PAC profile versus those meeting the aerobic-only guideline, and a more favorable aerobic-specific M-PAC profile versus those meeting the strength-only guideline. Finally we expected large differences in the aerobic and strength specific MPAC profiles of those meeting the aerobic-only versus strength-only guideline.

\section{Methods}

The design and methods of our survey study have been detailed elsewhere [7]. Briefly, ethical approval and informed consent were obtained for all procedures performed in the study. A stratified random sample of 2100 adult HCS (700 of each leukemia, Hodgkin lymphoma, and non-Hodgkin lymphoma) was contacted by the Alberta Cancer Registry to participate in this study. Data was collected from self-report questionnaires, where participants completed surveys by hand and returned them via post.

\section{Measures}

\section{Demographic and cancer-specific variables}

Demographic variables included age, sex, marital status, education, employment status, ethnicity, height, and weight. Cancer-specific variables included cancer type, previous treatments, time-since-diagnosis, current treatment status, cancer recurrence, current cancer status (existing disease versus disease-free), comorbidities, and whether participants received an exercise recommendation by one of their healthcare professionals involved in their cancer treatment.

\section{Aerobic and strength exercise behavior}

A modified version of the Godin Leisure Time Exercise Questionnaire (GLTEQ) was used to measure exercise behavior [11]. As the original GLTEQ did not separate aerobic and strength exercise, we included separate aerobic and strength questions. Participants were asked to first indicate the frequency and duration of any light, moderate, and vigorous aerobic exercise (i.e., exercise that improves the heart and lungs such as walking or running), they would have completed in a typical week over the past month. Participants were then asked to indicate the average frequency (days/week) and duration (minutes/session) of any moderate-to-intense strength exercise (i.e., exercise that improves muscular strength such as weight lifting, resistance bands, sit-ups, pushups) that they performed in a typical week over the past month. Weekly moderate-to-vigorous aerobic minutes 
were totaled, with vigorous minutes double weighted. Exercise levels were then dichotomized according to their respective guideline (i.e., aerobic: $<$ or $\geq 150 \mathrm{~min}$; strength: $<$ or $\geq 2$ sessions per week) [3]. Based on this information, we created a composite exercise guideline variable which categorized each HCS as meeting one of the following guidelines: neither, aerobic-only, strengthonly, or combined.

\section{Aerobic and strength exercise intention}

In line with the M-PAC, the decision to form an exercise intention was measured using two separate dichotomous items, one reflecting an intention to meet the aerobic guideline and the other to meet the strength guideline (i.e., "Do you intend to do regular aerobic/strength exercise over the next month? Yes/No") [12]. The term "regular exercise" was defined to reflect the levels of exercise that would constitute either the aerobic or strength guideline respectively (i.e., aerobic: $\geq 150$ weekly minutes; strength: $\geq 2$ weekly sessions). Again, we used this information to create another composite variable which categorized each HCS as either having an intention to meet the following guideline: neither, aerobic, strength, or combined.

\section{Motivational processes}

All of the following questionnaire items were first asked in relation to aerobic exercise and then repeated for strength exercise in a separate section of the questionnaire. Standard measures from the theory of planned behavior (TPB) assessed survivors' exercise motivation on a 7-point bipolar Likert scale [13, 14]. Six items captured attitude (e.g., useful-useless, enjoyable-unenjoyable). Three items measured injunctive norm (e.g., “... people who are important to me will be..." encouragingdiscouraging), and three items captured descriptive norm (e.g., "... people who are important to me will perform..." regular aerobic/strength exercise-no aerobic/strength exercise). Three items measured perceived control (e.g., “... regular aerobic/strength exercise over the next month would be completely up to me ..." strongly agree-disagree).

\section{Behavioral regulations}

Five items assessed exercise plans (when, where, and what type) using a 7-point scale (i.e., no plans - detailed plans) [15].

\section{Reflective processes}

Two items measured anticipated regret (e.g., "If I do not engage in regular aerobic/strength exercise over the next month, I will feel regret.") on an 11-point scale (i.e., definitely no - definitely yes) [16]. Exercise obligation and regulation over alternative activities were assessed using seven items on a 10-point scale (i.e., completely true for me - not at all true for me) [17]. Three items assessed exercise obligation (e.g., "I feel obligated to do regular aerobic/strength exercise over the next month...") and four items measured self-regulation over alternative competing activities (e.g., "Compared to doing regular aerobic/strength exercise over the next month, there are other things I could do which would be more fun..."). Items for self-regulation over competing alternative activities were reverse scaled so that higher scores would reflect greater self-regulation over competing activities.

\section{Statistical analyses}

Factor structures for the motivational, regulatory, and reflective variables are presented elsewhere [7, 8]. Briefly, separate exploratory factor analyses yielded identical seven factor structures for aerobic- and strength-specific M-PAC based variables (i.e., planning, obligation/regret, attitude, self-regulation over alternatives, descriptive norm, injunctive norm, and perceived control). Attitude, descriptive norm, injunctive norm, perceived control, and planning scores ranged from 1 to 7 , self-regulation over alternatives ranged from 1 to 10, and obligation/regret scores ranged from 1 to 10.4 because this factor combined two anticipated regret items (measured on 111 scales) and 3 obligation items (measured from 1 to 10). Descriptive statistics were used to estimate the prevalence of guideline adherence and the magnitude of the I-B gap. Multivariate analyses of variances (MANOVA) and chi-square analyses were used to examine differences in demographic, cancer, and M-PAC based variables between the four levels of guideline adherence. Any demographic or cancer variables that emerged significant in chi-square analyses were entered into a multinomial logistic regression to assess post hoc differences among the four guideline groups. Post hoc pairwise comparisons were conducted to interpret significant between-group differences for the M-PAC based variables.

\section{Results}

Participant flow through the study and characteristics are presented elsewhere [7]. Briefly, 29\% of those contacted for this study returned a completed survey (606/ 2100). Excluding return-to-senders and deceased persons yielded a $32 \%$ response rate $(606 / 1921)$. Overall, 186 (31\%) participants were leukemia, 187 (31\%) Hodgkin lymphoma, and 233 (38\%) non-Hodgkin lymphoma survivors. Based on limited data from the cancer registry, responders did not significantly differ from nonresponders on age, sex, disease stage, and time since diagnosis, but were more likely to be non-Hodgkin lymphoma survivors $(p<.001)$ and to have received chemotherapy $(p=.017)$. 


\section{Prevalence of HCS intending and meeting the exercise guidelines}

Table 1 reports the prevalence of intending and meeting the combined and independent exercise guidelines, as well as the I-B gap. Overall, 22\% (134/606) of HCS met the combined guideline, $22 \%(133 / 606)$ met aerobiconly, $10 \%$ (58/606) met strength-only, and 46\% (281/ 606) met neither guideline. In terms of exercise intention, 51\% (312/606) intended to meet the combined guideline, 19\% (116/606) the aerobic-only, 7\% (41/606) the strength-only, and 23\% (137/606) neither guideline. In terms of the I-B gap, $40 \%(127 / 312)$ of HCS with an intention to meet the combined guideline, $44 \%(51 / 116)$ with an intention to meet the aerobic-only, 37\% (15/41) with an intention to meet the strength-only, and $90 \%$ (124/137) with an intention to meet neither guideline, acted in accordance with their intention.

\section{Correlates of meeting neither, aerobic, strength, or combined guidelines}

Results from the chi-square analyses relating demographic and cancer variables to the four guideline categories are reported in Tables 2 and 3. Significant univariate associations emerged for age, education, employment status, number of children living at home, cancer type, cancer status, and comorbidities. When entered into a multinomial logistic regression, only the associations with age $(p=.008)$, number of children living at home $(p=.010)$, and education $(p<.001)$ remained independently significant $\left(R^{2}=.17, p<.001\right)$. In terms of M-PAC profiles (Table 4), the MANOVA revealed significant main effects for each aerobic- and strength-specific motivational, regulatory, and reflective variable (all $p s<.001$ ).

Traditional comparisons between combined, aerobic, and strength versus neither guideline

The traditional post hoc comparisons of demographic and cancer variables from the multinomial regression are reported in Additional file 1: Table S1. Compared to HCS meeting neither guideline, those meeting the combined guideline were younger and more highly educated; HCS meeting the aerobic-only guideline were younger; and no demographic or cancer variables distinguished HCS meeting the strength-only guideline. The traditional post hoc comparisons of M-PAC based variables from the MANOVA are reported in Additional file 1: Table S2. Compared to HCS meeting neither guideline, those meeting the combined guideline had significantly more favorable aerobic- and strength-specific M-PAC profile on all measured variables. Those meeting the strength-only guideline also reported significantly greater values on all strength-specific variables versus those meeting neither guideline. HCS meeting the aerobic-only guideline had significantly greater aerobic-specific motivations, regulations, and reflective processes on all variables except for regulation over alternatives.

\section{Novel comparisons among combined, aerobic, and strength guidelines}

Table 5 reports the novel post hoc comparisons of demographic and cancer variables between the different guidelines from the multinomial regression. HCS were more likely to meet the combined guideline over the aerobic-only guideline if they had no children living at home. They were also more likely to meet the combined guideline over both the aerobic and strength-only guidelines if they had completed university. HCS with no children living at home were more likely to meet the strength-only guideline over the aerobic-only guideline. Table 6 reports the novel post hoc comparisons of MPAC based variables between the different guidelines from the MANOVA. HCS meeting the combined guideline reported significantly more favorable strength-specific scores than those meeting the aerobic guideline, and more favorable aerobic-specific scores than those meeting the strength guideline, on all motivations, regulations, and reflective processes. Aerobic-specific attitude, perceived control, obligation/regret, and regulation over alternatives were favored by HCS meeting the aerobic versus strength-only guideline. Strength-specific attitude, planning, obligation/regret, and regulation over alternatives were favored by HCS meeting the strength versus aerobic-only guideline. Finally, HCS meeting the combined guidelines reported significantly more favorable strength-specific perceived control, planning, and obligation/regret than those meeting the strength guideline, and more favorable aerobic-specific perceived control, planning, obligation/regret, and regulation over alternatives than those meeting the aerobic guideline.

Table 1 The intention-behavior relationship for meeting neither, aerobic-only, strength-only, or combined exercise guidelines

\begin{tabular}{|c|c|c|c|c|}
\hline \multirow[b]{2}{*}{ Intention } & \multicolumn{4}{|l|}{ Behavior } \\
\hline & Neither $(n=281)$ & Aerobic $(n=133)$ & Strength $(n=58)$ & Combined $(n=134)$ \\
\hline Neither $(n=137)$ & $124(90 \%)$ & $8(6 \%)$ & $4(3 \%)$ & $1(1 \%)$ \\
\hline Aerobic-only $(n=116)$ & $59(51 \%)$ & $51(44 \%)$ & $2(2 \%)$ & $4(3 \%)$ \\
\hline Strength-only $(n=41)$ & $24(58 \%)$ & $0(0 \%)$ & 15 (37\%) & $2(5 \%)$ \\
\hline Combined $(n=312)$ & $74(24 \%)$ & $74(24 \%)$ & $37(12 \%)$ & $127(40 \%)$ \\
\hline
\end{tabular}


Table 2 Demographic profile of survivors meeting neither, aerobic-only, strength-only, or combined exercise guidelines

\begin{tabular}{|c|c|c|c|c|c|}
\hline Variable & Neither $(n=281)$ & Aerobic $(n=133)$ & Strength $(n=58)$ & Combined $(n=134)$ & $p$ value \\
\hline Age & & & & & $<.001$ \\
\hline$<60$ years $(n=303)$ & 99 (33\%) & $84(28 \%)$ & $28(9 \%)$ & $92(30 \%)$ & \\
\hline$\geq 60$ years $(n=303)$ & $182(60 \%)$ & $49(16 \%)$ & $30(10 \%)$ & $42(14 \%)$ & \\
\hline Gender & & & & & .22 \\
\hline Female $(n=341)$ & $147(43 \%)$ & $81(24 \%)$ & $31(9 \%)$ & $82(24 \%)$ & \\
\hline Male $(n=265)$ & $134(51 \%)$ & $52(20 \%)$ & $27(10 \%)$ & $52(20 \%)$ & \\
\hline Body Mass Index & & & & & .14 \\
\hline Normal weight $(n=221)$ & $96(43 \%)$ & $50(23 \%)$ & $19(9 \%)$ & $56(25 \%)$ & \\
\hline Overweight $(n=245)$ & $106(43 \%)$ & $57(23 \%)$ & $25(10 \%)$ & $57(23 \%)$ & \\
\hline Obese $(n=140)$ & $79(56 \%)$ & $26(19 \%)$ & $14(10 \%)$ & $21(15 \%)$ & \\
\hline Marital status & & & & & .46 \\
\hline Not married $(n=179)$ & $80(45 \%)$ & $36(20 \%)$ & $22(12 \%)$ & $41(23 \%)$ & \\
\hline Married $(n=427)$ & $201(47 \%)$ & $97(23 \%)$ & $36(8 \%)$ & $93(22 \%)$ & \\
\hline Children living at home & & & & & $<.001$ \\
\hline None $(n=450)$ & $222(49 \%)$ & $82(18 \%)$ & 49 (11\%) & $97(22 \%)$ & \\
\hline One or more $(n=156)$ & $59(38 \%)$ & $51(33 \%)$ & $9(6 \%)$ & $37(24 \%)$ & \\
\hline Education & & & & & $<.001$ \\
\hline University not completed $(n=295)$ & $159(54 \%)$ & $60(20 \%)$ & $36(12 \%)$ & $40(14 \%)$ & \\
\hline Completed university $(n=311)$ & $122(39 \%)$ & $73(24 \%)$ & $22(7 \%)$ & $94(30 \%)$ & \\
\hline Employment status & & & & & $<.001$ \\
\hline Not retired $(n=375)$ & $144(38 \%)$ & $91(24 \%)$ & $35(9 \%)$ & $105(28 \%)$ & \\
\hline Retired $(n=231)$ & $137(59 \%)$ & $42(18 \%)$ & $23(10 \%)$ & $29(13 \%)$ & \\
\hline Ethnicity & & & & & .14 \\
\hline White $(n=562)$ & $267(48 \%)$ & $123(22 \%)$ & $53(9 \%)$ & $119(21 \%)$ & \\
\hline Other $(n=44)$ & $14(32 \%)$ & $10(23 \%)$ & $5(11 \%)$ & $15(34 \%)$ & \\
\hline
\end{tabular}

\section{Discussion}

The purpose of this investigation was to estimate how many HCS met the combined, aerobic-only, strengthonly, and neither exercise guideline, and to examine what differentiates these four exercise groups. We previously reported in our two separate papers that $44 \%$ of HCS met the aerobic and 32\% met the strength guideline $[7,8]$. Our new results demonstrate that only $22 \%$ of HCS in our sample met the combined guidelines, $22 \%$ aerobic, $10 \%$ strength, and $46 \%$ neither guideline. These current results address a key limitation of our prior findings, and other studies examining aerobic and strength exercise separately, by accounting for the contamination that exists in a binary grouping scheme. For example, when examining the correlates of meeting the aerobic guideline separately, some of those categorized as meeting the aerobic guideline were in fact meeting the combined guideline, and some of those categorized as not meeting the aerobic guideline were in fact meeting the strength guideline. Thus, categorizing exercise guideline adherence into four categories avoids such confound, which also has implications for quantifying the I-B gap.

Specifically, the current investigation highlights that only about $40 \%$ of HCS who intended to meet either the aerobic, strength, or combined guideline followed-through on their intention, whereas our previous separate reports indicated that $60 \%$ of HCS successfully translated their aerobic exercise intention and 50\% realized their strength exercise intention $[7,8]$. Not only do we contend that the current results depict a more accurate illustration of the true I-B gap for HCS, but noting that almost no survivors (2\%) met the combined guideline without an intention to do so, supports a common criticism of the intention construct: that an intention is necessary but rarely dictates behavior alone [18-20]. Furthermore, HCS who intended to meet the combined guideline, rather than just the aerobic or strength guideline, were more likely to meet at least one of these exercise guidelines. So it appears that a necessary first step towards helping survivors meet the combined guideline is to aid their formation of an intention to 
Table 3 Cancer-specific profile of survivors meeting neither, aerobic-only, strength-only, or combined exercise guidelines

\begin{tabular}{|c|c|c|c|c|c|}
\hline Variable & Neither $(n=281)$ & Aerobic $(n=133)$ & Strength $(n=58)$ & Combined $(n=134)$ & $p$ value \\
\hline Cancer type & & & & & .008 \\
\hline Leukemia $(n=186)$ & $96(52 \%)$ & $37(20 \%)$ & $17(9 \%)$ & $36(19 \%)$ & \\
\hline Hodgkin lymphoma $(n=187)$ & $64(34 \%)$ & $47(25 \%)$ & $21(11 \%)$ & 55 (29\%) & \\
\hline non-Hodgkin lymphoma $(n=233)$ & $121(52 \%)$ & $49(21 \%)$ & $20(9 \%)$ & $43(19 \%)$ & \\
\hline Time since diagnosis & & & & & .11 \\
\hline$<2$ years $(n=116)$ & $53(46 \%)$ & $19(16 \%)$ & $17(15 \%)$ & $27(23 \%)$ & \\
\hline $2-5$ years $(n=304)$ & $137(45 \%)$ & $78(26 \%)$ & $21(7 \%)$ & $68(22 \%)$ & \\
\hline$>5$ years $(n=186)$ & $91(49 \%)$ & $36(19 \%)$ & $20(11 \%)$ & $39(21 \%)$ & \\
\hline Radiation & & & & & .27 \\
\hline No $(n=399)$ & $196(49 \%)$ & $83(21 \%)$ & $38(9 \%)$ & $82(21 \%)$ & \\
\hline Yes $(n=207)$ & $85(41 \%)$ & $50(24 \%)$ & $20(10 \%)$ & $52(25 \%)$ & \\
\hline Chemotherapy & & & & & .64 \\
\hline No $(n=173)$ & $81(47 \%)$ & $38(22 \%)$ & $20(12 \%)$ & $34(20 \%)$ & \\
\hline Yes $(n=433)$ & $200(46 \%)$ & $95(22 \%)$ & $38(9 \%)$ & $100(23 \%)$ & \\
\hline Stem cell/marrow transplant & & & & & .35 \\
\hline No $(n=541)$ & $255(47 \%)$ & $121(22 \%)$ & $50(9 \%)$ & $115(21 \%)$ & \\
\hline Yes $(n=65)$ & $26(40 \%)$ & $12(19 \%)$ & $8(12 \%)$ & $19(29 \%)$ & \\
\hline Treatment status & & & & & .13 \\
\hline Receiving treatments $(n=193)$ & 99 (51\%) & $38(20 \%)$ & $22(11 \%)$ & $34(18 \%)$ & \\
\hline Completed treatments $(n=413)$ & $182(44 \%)$ & $95(23 \%)$ & $36(9 \%)$ & $100(24 \%)$ & \\
\hline Recurrence & & & & & .88 \\
\hline No $(n=524)$ & $242(46 \%)$ & $113(22 \%)$ & $54(10 \%)$ & $118(23 \%)$ & \\
\hline Yes $(n=82)$ & $39(48 \%)$ & $20(24 \%)$ & $7(9 \%)$ & $16(20 \%)$ & \\
\hline Current cancer status & & & & & .007 \\
\hline Disease free $(n=372)$ & $156(42 \%)$ & $88(24 \%)$ & $32(9 \%)$ & $96(26 \%)$ & \\
\hline Existing disease $(n=234)$ & $125(53 \%)$ & 45 (19\%) & $26(11 \%)$ & $38(16 \%)$ & \\
\hline Comorbidities & & & & & $<.001$ \\
\hline None $(n=221)$ & $72(33 \%)$ & $56(25 \%)$ & $25(11 \%)$ & $68(31 \%)$ & \\
\hline One $(n=151)$ & $61(40 \%)$ & $42(28 \%)$ & $12(8 \%)$ & $36(24 \%)$ & \\
\hline Two or more $(n=234)$ & $148(63 \%)$ & $35(15 \%)$ & $21(9 \%)$ & $30(13 \%)$ & \\
\hline Exercise recommendation & & & & & .07 \\
\hline No $(n=376)$ & 185 (49\%) & 86 (23\%) & $36(10 \%)$ & 69 (18\%) & \\
\hline Yes $(n=230)$ & 96 (42\%) & 47 (20\%) & $22(10 \%)$ & 65 (28\%) & \\
\hline
\end{tabular}

do both regular aerobic and strength exercise. Examining this data further, we see that HCS were twice as likely to fall short of their goal to meet the combined guideline because they failed to do enough strength exercise (24\%) versus failing to meet the aerobic requirement (12\%).

As expected, our results provided overall support for the traditional hypotheses that HCS meeting the combined, aerobic, or strength guideline would differ on key demographic, cancer, and M-PAC based variables, versus those meeting neither guideline. As commonly found in the general literature, age and education were important correlates of exercise versus no exercise [21]. Specifically, age and education status differentiated HCS meeting the combined guideline from those meeting neither, and education differentiated HCS meeting the aerobic-only guideline from those meeting neither. Interestingly, no demographic or cancer variables distinguished HCS meeting the strength guideline from those meeting neither guideline, which may suggest that M-PAC based variables may be of greater importance for driving strength exercise behavior [7]. In terms of M-PAC profile differences, compared to HCS meeting neither guideline, those meeting the combined guideline had more favorable aerobic- and strength-specific M-PAC profiles, and those meeting the 
Table 4 Motivations, regulations, and reflective processes of survivors meeting neither, aerobic-only, strength-only, or combined exercise guidelines

\begin{tabular}{|c|c|c|c|c|}
\hline Variable & Neither $(n=281)$ & Aerobic $(n=133)$ & Strength $(n=58)$ & Combined $(n=134)$ \\
\hline \multicolumn{5}{|c|}{ Motivational processes } \\
\hline \multicolumn{5}{|l|}{ Attitude } \\
\hline Aerobic & $4.8(1.4)$ & $5.8(0.8)$ & $5.1(1.3)$ & $6.1(0.7)$ \\
\hline Strength & $4.4(1.5)$ & $5.0(1.2)$ & $5.6(1.1)$ & $6.0(0.7)$ \\
\hline \multicolumn{5}{|c|}{ Injunctive norm } \\
\hline Aerobic & $5.5(1.4)$ & $6.1(1.1)$ & $5.7(1.3)$ & $6.3(0.7)$ \\
\hline Strength & $5.2(1.6)$ & $5.5(1.4)$ & $5.7(1.4)$ & $6.1(0.9)$ \\
\hline \multicolumn{5}{|c|}{ Descriptive norm } \\
\hline Aerobic & $4.2(1.8)$ & $4.9(1.5)$ & $4.6(1.8)$ & $5.1(1.5)$ \\
\hline Strength & $3.6(1.8)$ & $3.9(1.9)$ & $4.3(1.8)$ & $4.3(1.7)$ \\
\hline \multicolumn{5}{|c|}{ Perceived control } \\
\hline Aerobic & $5.1(1.8)$ & $6.0(1.1)$ & $5.5(1.4)$ & $6.5(0.6)$ \\
\hline Strength & $5.1(1.9)$ & $5.7(1.5)$ & $5.8(1.2)$ & $6.3(0.9)$ \\
\hline \multicolumn{5}{|c|}{ Behavioral regulations } \\
\hline \multicolumn{5}{|l|}{ Planning } \\
\hline Aerobic & $2.3(1.9)$ & $3.4(2.2)$ & $4.5(1.9)$ & $5.4(1.5)$ \\
\hline Strength & $2.4(1.9)$ & $3.5(2.3)$ & $4.7(2.1)$ & $5.6(1.6)$ \\
\hline \multicolumn{5}{|c|}{ Reflective processes } \\
\hline \multicolumn{5}{|c|}{ Obligation/Regret } \\
\hline Aerobic & $4.6(3.0)$ & $7.9(2.1)$ & $5.5(3.0)$ & $8.7(1.7)$ \\
\hline Strength & $3.9(3.0)$ & $5.0(3.0)$ & $6.8(2.7)$ & $8.0(2.3)$ \\
\hline \multicolumn{5}{|c|}{ Regulation of alternatives } \\
\hline Aerobic & $5.5(2.6)$ & $6.0(2.4)$ & $5.1(2.2)$ & $6.8(2.3)$ \\
\hline Strength & $4.4(2.6)$ & $4.2(2.4)$ & $5.7(2.0)$ & $6.2(2.4)$ \\
\hline
\end{tabular}

Note. Standard deviations are presented in brackets. Post hoc comparisons are made between meeting the different guidelines. Attitudes, injunctive norms, descriptive norms, perceived control, and planning ranged from 1 to 7 , obligation/regret ranged from 1 to 10.4 , and regulation of alternatives ranged from 1 to 10

strength guidelines reported an overall more favorable strength-specific profile. Interestingly, HCS meeting the aerobic guideline reported a similar trend for aerobic-specific M-PAC based variables, however, their reported level self-regulation over alternative activities did not differ significantly from those meeting neither guideline. We speculate that this may reflect a unique facet of aerobic exercise which allows individuals to multi-task while participating. So, sacrificing one's involvement in competing activities (e.g., television watching) in order to exercise may not be required if multiple aims can be pursued simultaneously [22].

Our novel hypotheses compared HCS meeting the combined guideline, the aerobic-only guideline, and the strength-only guideline. HCS who completed university were twice as likely to meet the combined guideline over the aerobic-only guideline and three times as likely over the strength-only guideline. Thus, completing university may be associated with a greater awareness of the benefits of doing both regular aerobic and strength exercise, or may relate to better access to necessary resources (i.e., equipment, facilities) [21, 23]. Furthermore, HCS with no children living at home were significantly more likely to meet the combined guideline (two times) and strength-only guideline (three times) than the aerobiconly guideline. Exercise correlates research suggests that not having to care for dependents at home may alleviate some exercise-related time constraints [24, 25], but why this is more important for doing strength exercise over aerobic is unclear, especially when considering that the strength guideline can be satisfied in less overall time than the aerobic guideline.

As hypothesized, HCS meeting the combined guideline reported more favorable ratings on all strength-specific MPAC based variables than those meeting the aerobic-only guideline, and the same was true when comparing all aerobic-specific variables versus those meeting the strength guideline. The most intriguing finding from our novel comparisons, however, is that HCS meeting the combined guidelines reported significantly greater aerobic-specific 
Table 5 Multinomial regression comparing the demographic and cancer-specific correlates between the combined, aerobic-only, and strength-only guidelines

\begin{tabular}{|c|c|c|c|c|c|c|}
\hline \multirow[b]{2}{*}{ Variable } & \multicolumn{2}{|c|}{ Combined vs Aerobic } & \multicolumn{2}{|c|}{ Combined vs Strength } & \multicolumn{2}{|c|}{ Strength vs Aerobic } \\
\hline & OR $(95 \% \mathrm{Cl})$ & $p$ & OR $(95 \% \mathrm{Cl})$ & $p$ & OR $(95 \% \mathrm{Cl})$ & $p$ \\
\hline \multicolumn{7}{|l|}{ Age } \\
\hline$<60$ years vs $\geq 60$ years & $0.8(0.4-1.9)$ & .79 & $1.6(0.6-3.9)$ & .35 & $0.6(0.2-1.5)$ & .25 \\
\hline \multicolumn{7}{|l|}{ Children living at home } \\
\hline None vs. One or more & $2.2(1.2-3.9)$ & .006 & $0.7(0.3-1.7)$ & .46 & $3.1(1.3-7.2)$ & .010 \\
\hline \multicolumn{7}{|l|}{ Education } \\
\hline University completed vs. not completed & $2.0(1.2-3.4)$ & .008 & $3.4(1.7-6.6)$ & $<.001$ & $0.6(0.3-1.1)$ & .11 \\
\hline \multicolumn{7}{|l|}{ Employment status } \\
\hline Not retired vs. Retired & $1.7(0.8-3.8)$ & .16 & $1.3(0.5-3.2)$ & .60 & $1.4(0.6-3.4)$ & .50 \\
\hline \multicolumn{7}{|l|}{ Cancer type } \\
\hline Leukemia \& non-Hodgkin lymphoma vs. Hodgkin lymphoma & $1.1(0.6-1.9)$ & .74 & $0.8(0.4-1.6)$ & .47 & $1.4(0.7-3.0)$ & .33 \\
\hline \multicolumn{7}{|l|}{ Current cancer status } \\
\hline Disease free vs. Existing disease & $1.1(0.6-1.9)$ & .78 & $1.8(0.9-3.8)$ & .10 & $0.6(0.3-1.2)$ & .15 \\
\hline \multicolumn{7}{|l|}{ Comorbidities } \\
\hline None vs. one or more & $1.4(0.8-2.4)$ & .27 & $0.9(0.4-1.9)$ & .78 & $1.5(0.7-3.1)$ & .26 \\
\hline
\end{tabular}

Note. OR odds ratio, $\mathrm{Cl}$ confidence interval. All comparisons are in reference to the second listed group in each dyad

ratings of perceived control, planning, and obligation/regret than those meeting the aerobic-only guideline. Furthermore, this identical trend resulted for strength-specific ratings versus those meeting the strength-only guideline. Thus, these results suggest that efforts targeted towards helping HCS meet the combined guideline when already adhering to one guideline should focus on promoting both exercise modalities and not just the one in which they are deficient. For example, significant improvements in aerobic-specific perceived control, regulations, and reflective processes may help HCS meet the combined guideline, even if they already meet the aerobic guideline. Thus, we may need to reconsider the intuitive approach of only promoting the motivations, regulations, and reflective processes for the "deficient guideline," and consider the additional need to promote (or "top-up") the currently "performed guideline." Altogether, these results speak to the overall benefit of using action control models (such as the M-PAC) [26], as the majority of the differentiating features between HCS meeting the combined guideline versus either singular guideline were behavioral regulations and reflective processes that are not typically captured in more traditional models (such as the TPB) [27].

Our study has important strengths and limitations. The strengths of our study include being one of the few to quantify adherence to the four categories of the exercise guidelines, the first to examine the I-B gap and M-PAC correlates in such a context, one of the few to examine the correlates of exercise in HCS, the large population-based sample of HCS, and the validated measures for social cognitive variables specific to both aerobic and strength exercise. The limitations of this study include a potential self-selection sample bias, the use of self-report measures of exercise, the cross-sectional design, and not measuring additional potentially important variables.

Our sample may have been biased due to selfselection. Though the HCS who completed the survey were not significantly different than non-respondents in age, sex, disease stage, and time since diagnosis, they likely had more favorable exercise-specific M-PAC profiles, as well as higher rates of exercise intention and participation. These biases may not only have affected our estimates of the prevalence of exercise intentions and behavior but also their associations with the correlates of meeting the exercise guidelines. Therefore, it is unclear if our findings generalize to the broader population of less motivated and active HCS. The use of selfreported measures could be influenced by recall and reporting biases which may have prompted participants to over-report their actual levels of exercise participation and motivation. Furthermore, to date, no validated or sufficiently detailed self-report measure of strength exercise exists, and we are therefore unable to comment on the quality of participants' strength training programs. By employing a cross-sectional design, we were unable to examine the causal sequencing or hierarchy of variables in relation to participants' exercise levels. Finally, our survey did not assess other potentially important variables such as participants' knowledge of the exercise guidelines, their exercise history before diagnosis, and exercise habits. These variables could influence the 
Table 6 Pairwise comparisons of exercise-specific motivations, regulations, and reflective processes, between combined, aerobiconly, and strength-only guidelines

\begin{tabular}{|c|c|c|c|}
\hline Variable & Combined vs Aerobic & Combined vs Strength & Aerobic vs Strength \\
\hline \multicolumn{4}{|c|}{ Motivational processes } \\
\hline \multicolumn{4}{|l|}{ Attitude } \\
\hline Aerobic & $p=.07, d=0.35$ & $p<.001, d=0.90$ & $p<.001, d=0.63^{\mathrm{a}}$ \\
\hline Strength & $p<.001, d=0.98$ & $p=.05, d=0.41$ & $p=.004, d=0.50^{\mathrm{b}}$ \\
\hline \multicolumn{4}{|c|}{ Injunctive norm } \\
\hline Aerobic & $p=.15, d=0.24$ & $p=.002, d=0.57$ & $p=.05, d=0.32^{\mathrm{a}}$ \\
\hline Strength & $p<.001, d=0.53$ & $p=.07, d=0.35$ & $p=.31, d=0.16^{b}$ \\
\hline \multicolumn{4}{|c|}{ Descriptive norm } \\
\hline Aerobic & $p=.25, d=0.16$ & $p=.042, d=0.32$ & $p=.26, d=0.18^{\mathrm{a}}$ \\
\hline Strength & $p=.05, d=0.24$ & $p=.89, d=0.02$ & $p=.17, d=0.21^{b}$ \\
\hline \multicolumn{4}{|c|}{ Perceived control } \\
\hline Aerobic & $p=.010, d=0.50$ & $p<.001, d=0.92$ & $p=.012, d=0.44^{a}$ \\
\hline Strength & $p<.001, d=0.56$ & $p=.042, d=0.49$ & $p=.48, d=0.13^{b}$ \\
\hline \multicolumn{4}{|c|}{ Behavioral regulations } \\
\hline \multicolumn{4}{|l|}{ Planning } \\
\hline Aerobic & $p<.001, d=1.02$ & $p=.005, d=0.49$ & $p<.001, d=0.52^{\mathrm{b}}$ \\
\hline Strength & $p<.001, d=1.06$ & $p=.003, d=0.50$ & $p<.001, d=0.54^{b}$ \\
\hline \multicolumn{4}{|c|}{ Reflective processes } \\
\hline \multicolumn{4}{|c|}{ Obligation/Regret } \\
\hline Aerobic & $p=.013, d=0.42$ & $p<.001, d=1.30$ & $p<.001, d=0.93^{a}$ \\
\hline Strength & $p<.001, d=1.10$ & $p=.011, d=0.45$ & $p<.001, d=0.62^{b}$ \\
\hline \multicolumn{4}{|c|}{ Regulation of alternatives } \\
\hline Aerobic & $p=.012, d=0.33$ & $p<.001, d=0.75$ & $p=.016, d=0.40^{\mathrm{a}}$ \\
\hline Strength & $p<.001, d=0.86$ & $p=.18, d=0.23$ & $p<.001, d=0.70^{b}$ \\
\hline
\end{tabular}

Note. ${ }^{a}=$ comparisons favoring the aerobic-only guideline group, ${ }^{b}=$ comparisons favoring the strength-only group. Hypothesized comparisons are bolded

likelihood of HCS meeting the exercise guidelines and could have been used to additionally discern whether survivors were recent exercise adopters or long-term maintainers.

\section{Conclusions}

In conclusion, we took a novel approach to examining the correlates of exercise behavior by simultaneously analyzing the aerobic and strength exercise guidelines. Our results revealed that $22 \%$ of HCS met the combined exercise guideline, $22 \%$ aerobic-only, $10 \%$ strength-only, and $46 \%$ met neither guideline. Having no children living at home and more formal education emerged as important correlates of meeting the combined over the aerobicor strength-only guideline. HCS meeting the combined guideline also reported more favorable ratings on all strength-specific M-PAC based variables than those meeting the aerobic-only guideline, and all aerobicspecific variables than those meeting the strength guideline. To help HCS meet the combined guidelines, it appears important to promote increased motivations, regulations, and reflective processes for both types of exercise including the exercise guideline that they are already meeting. These results may be helpful for designing health-promotion interventions aimed at helping HCS meet the aerobic and strength guidelines, thereby optimizing health outcomes.

\section{Additional file}

Additional file 1: Table S1. Multinomial regression of demographic and cancer-specific correlates comparing combined, aerobic-only, and strength-only guidelines versus neither. Table S2. Pairwise comparisons of exercise-specific motivations, regulations, and reflective processes versus neither guideline. (DOCX $22 \mathrm{~kb}$ )

\section{Acknowledgements}

Not applicable.

\section{Funding}

JRV is supported by the Frederick Banting and Charles Best Canada Graduate Scholarship (CGS-D) through the Canadian Institutes of Health Research. RER is supported by a Canadian Cancer Society Senior Scientist Award and the Right to Give Foundation. KSC is supported by the Canada Research Chairs Program. 


\section{Availability of data and materials}

The data that support the findings of this study are available from the corresponding author upon reasonable request.

\section{Authors' contributions}

JRV and KSC made substantial contributions to conception and design, acquisition of data, analysis and interpretation of data, drafting of the manuscript, revising the manuscript, and gave final approval for publication. RER and GJW made substantial contributions to conception and design, analysis and interpretation of data, revising the manuscript, and gave final approval for publication.

\section{Competing interests}

The authors declare that they have no competing interests.

\section{Consent for publication}

Not applicable.

\section{Ethics approval and consent to participate}

Ethics approval was granted by the Health Research Ethics Board of Alberta (HREBA) Cancer Committee (HREBA.CC-14-0097).

\section{Publisher's Note}

Springer Nature remains neutral with regard to jurisdictional claims in published maps and institutional affiliations.

\section{Author details}

${ }^{1}$ Faculty of Physical Education and Recreation, University of Alberta, Edmonton, AB, Canada. ${ }^{2}$ School of Exercise Science, Physical \& Health Education, University of Victoria, Victoria, BC, Canada.

Received: 26 January 2017 Accepted: 20 March 2017

\section{Published online: 28 March 2017}

\section{References}

1. Mishra SI, Scherer RW, Geigle PM, Berlanstein DR, Topaloglu O, Gotay CC, Snyder C. Exercise interventions on health-related quality of life for cancer survivors. Cochrane Database Syst Rev. 2012;8:CD007566.

2. Strasser B, Steindorf K, Wiskemann J, Ulrich CM. Impact of resistance training in cancer survivors: a meta-analysis. Med Sci Sports Exerc. 2013;45:2080-90.

3. Schmitz KH, Courneya KS, Matthews C, Demark-Wahnefried W, Galvao DA, Pinto BM, Irwin ML, Wolin KY, Segal RJ, Lucia A, et al. American College of Sports Medicine roundtable on exercise guidelines for cancer survivors. Med Sci Sports Exerc. 2010:42:1409-26.

4. Forbes CC, Blanchard CM, Mummery WK, Courneya KS. A comparison of physical activity correlates across breast, prostate and colorectal cancer survivors in Nova Scotia, Canada. Support Care Cancer. 2014;22:891-903.

5. Forbes CC, Blanchard CM, Mummery WK, Courneya KS. Prevalence and correlates of strength exercise among breast, prostate, and colorectal cancer survivors. Oncol Nurs Forum. 2015:42:118-27.

6. Crawford JJ, Holt NL, Vallance JK, Courneya KS. A new paradigm for examining the correlates of aerobic, strength, and combined exercise: an application to gynecologic cancer survivors. Support Care Cancer. 2016;24: 3533-41.

7. Vallerand JR, Rhodes RE, Walker GJ, Courneya KS. Understanding strength exercise intentions and behavior in hematologic cancer survivors: an analysis of the intention-behavior gap. J Cancer Surviv. 2016;10:945-55.

8. Vallerand JR, Rhodes RE, Walker GJ, Courneya KS. Explaining the aerobic exercise intention-behavior gap in cancer survivors. Am J Health Behav. 2016:40:675-84.

9. Rhodes RE, De Bruijn GJ. What predicts intention-behavior discordance? a review of the action control framework. Exerc Sport Sci Rev. 2013;41:201-7.

10. Rhodes RE. The evolving understanding of physical activity behavior: a multi-process action control approach. Adv Motivation Sci. in press.

11. Godin G, Shephard RJ. A simple method to assess exercise behavior in the community. Can J Appl Sport Sci. 1985;10:141-6.

12. Courneya KS. Predicting repeated behavior from intention: the issue of scale correspondence. J Appl Soc Psychol. 1994;24:580-94.

13. Aizen, I. Constructing a theory of planned behavior questionnaire. 2006. http://www-unix.oit.umass.edu/ aizen/pdf/tpb.measurement.pdf. Accessed 14 Jan 2017.
14. Rhodes RE, Blanchard CM, Matheson DH. A multicomponent model of the theory of planned behaviour. Br J Health Psychol. 2006;11:119-37.

15. Luszczynska A, Schwarzer R. Planning and self-efficacy in the adoption and maintenance of breast self-examination: a longitudinal study on selfregulatory cognitions. Psychol Health. 2003;18:93-108.

16. Abraham C, Sheeran P. Acting on intentions: the role of anticipated regret. Br J Soc Psychol. 2003;42:495-511.

17. Wilson PM, Rodgers WM, Carpenter PJ, Hall C, Hardy J, Fraser SN. The relationship between commitment and exercise behavior. Psychol Sport Exerc. 2004;5:405-21.

18. Sheeran P. Intention — behavior relations: a conceptual and empirical review. Eur Rev Soc Psychol. 2002;12:1-36.

19. Sniehotta FF, Presseau J, Araujo-Soares V. Time to retire the theory of planned behaviour. Health Psychol Rev. 2014;8:1-7.

20. Rhodes RE, De Bruijn GJ. How big is the physical activity intentionbehaviour gap? A meta-analysis using the action control framework. Br J Health Psychol. 2013;18:296-309.

21. Bauman AE, Reis RS, Sallis JF, Wells JC, Loos RJF, Martin BW. Correlates of physical activity: why are some people physically active and others not? Lancet. 2012;380:258-71.

22. Rhodes RE, Quinlan A, Mistry CD. Do other goals influence physical activity? A systematic review examining the relationship between other goals and physical activity behavior. Prev Med. 2016;91:306-17.

23. Sander AP, Wilson J, Izzo N, Mountford SA, Hayes KW. Factors that affect decisions about physical activity and exercise in survivors of breast cancer: a qualitative study. Phys Ther. 2012;92:525-36.

24. Ottenbacher AJ, Day RS, Taylor WC, Sharma SV, Sloane R, Snyder DC, Kraus WE, Demark-Wahnefried W. Exercise among breast and prostate cancer survivors-what are their barriers? J Cancer Surviv. 2011;5:413-9.

25. Bellows-Riecken KH, Rhodes RE. A birth of inactivity? A review of physical activity and parenthood. Prev Med. 2008:46:99-110.

26. Rhodes RE, Yao CA. Models accounting for intention-behavior discordance in the physical activity domain: a user's guide, content overview, and review of current evidence. Int J Behav Nutr Phys Act. 2015;12:9.

27. Ajzen I. The theory of planned behavior. Organ Behav Hum Decis Process. 1991;50:179-211.

\section{Submit your next manuscript to BioMed Central and we will help you at every step:}

- We accept pre-submission inquiries

- Our selector tool helps you to find the most relevant journal

- We provide round the clock customer support

- Convenient online submission

- Thorough peer review

- Inclusion in PubMed and all major indexing services

- Maximum visibility for your research

Submit your manuscript at www.biomedcentral.com/submit
Biomed Central 